\title{
Some Properties of Soft $\beta$-Connected Spaces in Soft Topological Spaces
}

\author{
S.S. Benchalli ${ }^{a}$, P.G. Patil ${ }^{b^{*}}$ and Abeda S. Dodamani ${ }^{\mathrm{c}}$ \\ Department of Mathematics, Karnatak University, Dharwad, 580003, India \\ abenchalliss@gmail.com, bpgpatil01@gmail.com*, 'mail2abeda@gmail.com
}

Keywords: Soft sets, Soft topological Spaces, Soft $\beta$-Connected Spaces, Soft $\beta$-Boundary

\begin{abstract}
Soft set theory is a newly emerging tool to deal with uncertain problems and has been studied by researchers in theory and practice. In this paper, we investigated the properties and characterizations of soft $\beta$-connected spaces in soft topological spaces. We anticipate that the results in this paper can be stimulated to the further study on soft topology to accomplish genenral framework for the practical life applications.
\end{abstract}

\section{Introduction}

The researchers introduced the concept of soft sets to deal with uncertainty and to solve complicated problems in economics, engineering, medicines, sociology and environment because of unsuccessful use of classical methods. The well-known theories that can be considered as mathematical tools for dealing with uncertainties and imperfect knowledge are: theory of fuzzy sets [1], theory of vague sets, theory of interval mathematics [2], theory of intuitionists fuzzy sets [3], theory of rough sets and theory of probability $[4,5]$. All these tools require the pre specification of some parameter to start with.

In 1999 Molodtsov [6] initiated the theory of soft sets as a new mathematical tool to deal with uncertainties while modeling the problems with incomplete information. In [7], he applied successfully in directions such as, smoothness of functions, game theory, operations research, Riemann-integration, Perron integration, probability and theory of measurement. Maji et al. [8, 9] gave first practical application of soft sets in decision making problems.

Shabir and Naz [10] initiated the study of soft topological spaces. They defined basic notions of soft topological spaces such as soft open, soft closed sets, soft subspace, soft closure, soft neighborhood of a point, soft Ti-spaces, for $\mathrm{i}=1 ; 2 ; 3 ; 4$, soft regular spaces, soft normal spaces and established their several properties. In $2011 \mathrm{~S}$. Hussain et al. [11] continued investigating the properties of soft open(closed), soft neighborhood and soft closure. They also defined and discussed the properties of soft interior, soft exterior and soft boundary. Also in the year 2012 B. Ahmad and S. Hussain [12] explored the structures of soft topology using soft points.

A. Kharral et al. [13] defined and discussed the many properties of soft images and soft inverse images of soft sets. They also applied these notions to the problem of medical diagnosis in medical systems. In [14] I. Zorlutana et al, defined and discussed soft pu-continuous mappings. M. Akdag et al. [15] have studied soft b-open sets and soft b-continuous functions, further V. Seenivasan et al. [16] introduced soft gsg-closed sets. I. Arockiarani et al. [17] studied the soft $\mathrm{g} \beta$-closed sets and soft gs $\beta$-closed sets in soft topological spaces. In [18] J. Subhashinin et al. have studied soft P-connectedness in soft topological spaces and Bin Chen [19] continued studying some properties of soft semi-open sets,

Recently S.S. Benchalli et al. [20-24] have studied soft $\beta$-separation axioms, soft $\beta$-compactness, soft $\beta$-locally closed spaces, soft regularity, soft normality, weaker forms of soft closed sets and soft $\gamma$-operations in soft topological spaces. In the present paper new concept in soft topological spaces such as soft $\beta$-connected space, soft $\beta$-boundary and some of their properties are discussed. 
The organization of this paper is as follows: Section 2 briefly reviews some basic concepts about soft sets, soft compactness and related properties in soft topological spaces; Section 3 defines the concepts of soft $\beta$-connected spaces and few definitions are given; Section 4 is the main results of the paper, which introduces the soft $\beta$-connected and soft $\beta$-notconnected spaces and relative properties are studied; Section 5 is conclusion of the paper.

\section{Preliminary}

Through-out this paper $(X, \tau, E)$ will be a soft topological space.

Definition 2.1. [10] Let $\mathrm{X}$ be an initial universe and let $\mathrm{E}$ be a set of parameters. Let $\mathrm{P}(\mathrm{X})$ denote the power set of $\widetilde{X}$ and let A be a nonempty subset of E. A pair $(F, A)$ is called a soft set over $\tilde{X}$, where $\mathrm{F}$ is a mapping given by $F: A \rightarrow P(X)$. In other words, a soft set over $\widetilde{X}$ is a parameterized family of subsets of the universe $\tilde{X}$. For $\varepsilon \in A, F(\varepsilon)$ may be considered as the set of $\varepsilon$-approximate elements of the soft set $(F, A)$. Clearly, a soft set is not a set.

Definition 2.2. [9] A soft set (F, E) over $X$ is said to be an absolute soft set, denoted by $\tilde{X}$, if for all $\mathrm{e} \in \mathrm{E}, F(e)=\tilde{X}$. Clearly $\tilde{X}^{c}=\phi_{E}$ and $\phi_{E}^{c}=\tilde{X}$.

Definition 2.3. [10] Let $\mathrm{Y}$ be a nonempty subset of $\tilde{X}$; then $\tilde{Y}$ denotes the soft set $(Y, E)$ over $\tilde{X}$ for which $Y(e)=Y$, for all $e \in E$.

Definition 2.4. [9] The union of two soft sets of $(F, A)$ and $(G, B)$ over the common universe $\tilde{X}$ is the soft set $(H, C)$, where $C=A \cup B$ and for all $e \in C$

$$
H(e)=\left\{\begin{array}{cc}
F(e) & \text { if } \mathrm{e} \in \mathrm{A}-\mathrm{B} \\
G(e) & \text { if } \mathrm{e} \in \mathrm{B}-\mathrm{A} \\
F(e) \cup G(e) & \text { if } \mathrm{e} \in \mathrm{A} \cap \mathrm{B}
\end{array}\right.
$$

We write $(F, A) \widetilde{\cup}(G, A)=(H, C)$.

Definition 2.5. [9] The intersection $(H, C)$ of two soft sets $(F, A)$ and $(G, B)$ over a common universe $\tilde{X}$, denoted by $(F, A) \cap(G, B)$, is defined as $C=A \cap B$, and $H(e)=F(e) \cap G(e)$ for all $e \in C$.

Definition 2.6. [9] Let $(F, A)$ and $(G, B)$ be two soft sets over a common universe $\tilde{X}$. $(F, A) \widetilde{\subset}(G, B)$, if $A \subset B$, and $H(e)=F(e) \subset G(e)$ for all $e \in A$.

Definition 2.7. [10] Let $\tau$ be the collection of soft sets over $\tilde{X}$; then $\tau$ is said to be a soft topology on $\tilde{X}$ if it satisfies the following axioms:

(1) $\Phi, \tilde{X}$ belong to $\tau$.

(2) The union of any number of soft sets in $\tau$ belongs to $\tau$.

(3) The intersection of any two soft sets in $\tau$ belongs to $\tau$.

The triplet $(X, \tau, E)$ is called a soft topological space over $\tilde{X}$. Then the members of $\tau$ are said to be soft open sets in $\tilde{X}$. The relative complement of a soft set $(F, A)$ is denoted by $(F, A)^{c}$ and is defined by $(F, A)^{c}=\left(F^{c}, A\right)$. 
Definition 2.8. [26] Let $(X, \tau, E)$ be a soft topological space over $\widetilde{X}$ and let $(F, A)$ be a soft set over X.

(1) The soft interior of $(F, A)$ is the soft set $\operatorname{int}((F, A))=\cup\{(O, A):(O, A)$ is soft open and $(O, A) \widetilde{\subset}(F, A)\}$.

(2) The soft closure of $(F, A)$ is the soft set $\operatorname{cl}((F, A))=\cap\{(F, E):(F, E)$ is soft closed and $(F, A) \widetilde{\subset}(F, E)\}$

Definition 2.9. [27] A soft set $(F, A)$ of a soft topological space $(X, \tau, E)$ is said to be

(a) Soft open if its complement is soft closed,

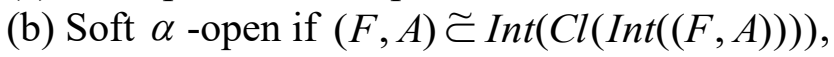

(c) Soft preopen if $(F, A) \widetilde{C} \operatorname{Int}(C l((F, A)))$,

(d) Soft semiopen if $(F, A) \widetilde{\subset} C l(\operatorname{Int}((F, A)))$,

(e) Soft $\beta$-open if $(F, A) \simeq C l(\operatorname{Int}(C l((F, A))))$.

The complement of soft open, (resp. soft $\alpha$-open, soft preopen, soft semiopen, soft $\beta$-open) sets are said to be soft closed (resp.soft $\alpha$-closed, soft preclosed, soft semiclosed, soft $\beta$-closed). The intersection of soft closed (resp. soft $\alpha$-closed, soft preclosed, soft semiclosed, soft $\beta$-closed) sets containing $(\mathrm{F}, \mathrm{A})$ is called the soft closure (resp. soft $\alpha$-closure, soft pre-closure, soft semiclosure, soft $\beta$-closure) of $(F, A)$ and is denoted by $\operatorname{scl}(F, A)$ (resp. $s \alpha \mathrm{Cl}(F, A), \operatorname{sPCl}(F, A)$, $\operatorname{sSCl}(F, A), \mathrm{s} \beta \mathrm{Cl}(F, A))$. The soft interior of $(F, A)$ is defined by the union of all soft open (resp.soft $\alpha$-open, soft preopen, soft semiopen, soft $\beta$-open ) sets contained in $(F, A)$ and is denoted by $\operatorname{sint}(F, A)($ resp.s $\alpha$ Int $(F, A), \operatorname{sPInt}(F, A), \operatorname{sSInt}(F, A), \mathrm{s} \beta \operatorname{Int}(F, A))$.

Theorem 2.10. [27] In any soft topological space, the following results hold:

(a) Every soft open set is soft $\alpha$-closed.

(b) Every soft $\alpha$-open set is soft preopen.

(c) Every soft $\alpha$-open set is soft semiopen.

(d) Every soft semiopen set is soft $\beta$-open.

(e) Every soft preclosed set is soft $\beta$-open.

Definition 2.11. [13] Let $S S(X)_{E}$ and $S S(Y)_{E}$ be families of soft sets. $u: X \rightarrow Y$ and $p: E \rightarrow E^{\prime}$ be mappings. Then a mapping $f_{p u}: S S(X)_{E} \rightarrow S S(Y)_{E^{\prime}}$ defined as:

(1) Let $(F, E)$ be a soft set in $S S(X)_{E}$. The image of $(F, E)$ under $f_{p u}$, written as $f_{p u}(F, E)=\left(f_{p u}(F), p(E)\right)$, is a soft set in $S S(Y)_{E}$ such that

$$
f_{p u}(F, E)=\left\{\begin{array}{cc}
\cup_{x \in p^{\prime}(y) \cap A} u(F(x)), & \mathrm{p}^{\prime}(\mathrm{y}) \cap \mathrm{A} \neq \phi \\
\phi, & \text { otherwise }
\end{array} \text { for all } y \in E^{\prime}\right.
$$

(2) Let $\left(G, E^{\prime}\right)$ be a soft set in $S S(V)_{E^{\prime}}$. Then the inverse image of $\left(G, E^{\prime}\right)$ under $f_{p u}$, written as $f_{p u}^{-1}\left(G, E^{\prime}\right)=\left(f_{p u}^{-1}(G) \cdot p^{-1}\left(E^{\prime}\right)\right)$, is a soft set in $S S(U)_{E}$ such that

$$
f_{p u}^{-1}\left(G, E^{\prime}\right)=\left\{\begin{array}{cc}
u^{-1}(G(p(x))), & \mathrm{p}(\mathrm{x}) \in \mathrm{E}^{\prime} \\
\phi, & \text { otherwise }
\end{array} \text { for all } x \in E .\right.
$$




\section{Soft $\beta$-Connected Spaces in Soft Topological Spaces}

In this section, the concepts of soft $\beta$-connected space, soft $\beta$-boundary and their properties are studied comprehensively.

Definition 3.1. Two soft $\beta$-open sets $(F, E)$ and $(G, E)$ over a common universe $\tilde{X}$ are soft $\beta$-disjoint, if $(F, E) \cap(G, E)=\phi$. That is $F(e) \cap G(e)=\phi$, for all $e \in E$.

Definition 3.2. Let $(\mathrm{X}, \tau, \mathrm{E})$ be a soft topological space, and $\left(F_{1}, E\right),\left(F_{2}, E\right)$ be two soft $\beta$-open sets over $\tilde{X}$. Then soft $\beta$-open sets $\left(F_{1}, E\right)$ and $\left(F_{2}, E\right)$ are said to be soft $\beta$-separated if $\left(F_{1}, E\right) \cap s \beta c l\left(F_{2}, E\right)=\phi$ and $s \beta c l\left(F_{1}, E\right) \cap\left(F_{2}, E\right)=\phi$.

Remark: Two disjoint soft $\beta$-open sets over $\widetilde{X}$ may not be soft $\beta$-separated.

Definition 3.3. Let $(X, \tau, E)$ be a soft topological space over $\tilde{X}$. Then $(X, \tau, E)$ is said to be soft $\beta$-connected, if there does not exist a pair (F, E) and (G, E) of non empty soft disjoint soft $\beta$-open subsets of $(X, \tau, E)$ such that $\widetilde{X}=(F, E) \cup(G, E)$, otherwise $(X, \tau, E)$ is said to be soft $\beta$-disconnected. In this case, the pair $(F, E)$ and $(G, E)$ is called the soft $\beta$-disconnection of $\tilde{X}$.

Example 3.4. Let $X=\left\{h_{1}, h_{2}, h_{3}\right\}$ and $E=\left\{e_{1}, e_{2}\right\}$ and

$$
(X, \tau, E)=\left\{\phi, \widetilde{X},\left(F_{1}, E\right),\left(F_{2}, E\right),\left(F_{3}, E\right),\left(F_{4}, E\right),\left(F_{5}, E\right)\right\},
$$

where $\left(F_{1}, E\right),\left(F_{2}, E\right),\left(F_{3}, E\right),\left(F_{4}, E\right),\left(F_{5}, E\right)$ are soft $\beta$ - open sets over $\tilde{X}$, defined as follows:

$$
\begin{aligned}
& \left(F_{1}, E\right)=\left\{\left(e_{1},\left\{h_{2}\right\}\right),\left(e_{2},\left\{h_{1}\right\}\right)\right\}, \\
& \left(F_{2}, E\right)=\left\{\left(e_{1},\left\{h_{2}, h_{3}\right\}\right),\left(e_{2},\left\{h_{1}, h_{2}\right\}\right)\right\}, \\
& \left(F_{3}, E\right)=\left\{\left(e_{1},\left\{h_{1}, h_{2}\right\}\right),\left\{\left(e_{2}, X\right)\right\},\right. \\
& \left(F_{4}, E\right)=\left\{\left(e_{1},\left\{h_{1}, h_{2}\right\}\right),\left(e_{2},\left\{h_{1}, h_{3}\right\}\right)\right\} \\
& \left(F_{5}, E\right)=\left\{\left(e_{1},\left\{h_{2}\right\}\right),\left(e_{2},\left\{h_{1}, h_{2}\right\}\right)\right\} .
\end{aligned}
$$

Then $(X, \tau, E)$ defines a soft topology on $\widetilde{X}$ and hence $(X, \tau, E)$ is a soft topological space over $\tilde{X}$. Clearly $\tilde{X}$ is soft $\beta$-connected.

\section{Results}

Theorem 4.1. Let $(\mathrm{X}, \tau, \mathrm{E})$ be a soft topological space. Then the following statements are equivalent:

(1) $\mathrm{X}$ is soft $\beta$-connected.

(2) $\mathrm{X}$ and $\phi$ are the only soft $\beta$-clopen sets in $(\mathrm{X}, \tau$,E).

(3) $(\mathrm{X}, \tau, \mathrm{E})$ is not the soft union of two soft disjoint non empty soft $\beta$-open sets.

(4) $(\mathrm{X}, \tau, \mathrm{E})$ is not the soft union of two soft disjoint non empty soft $\beta$-closed sets.

(5) $(\mathrm{X}, \tau, \mathrm{E})$ is not the soft union of two soft disjoint non empty soft $\beta$-separated sets.

Proof: $(1) \Rightarrow(2)$ By definition of soft $\beta$-connected space, the proof is obvious.

$(2) \Rightarrow(3)$

Suppose (3) is false and $(F, E)=(F, A) \cup(F, B)$, where $(\mathrm{F}, \mathrm{A})$ and $(\mathrm{F}, \mathrm{B})$ are soft disjoint non-empty $\beta$-open sets. Since $(F, E)-(F, A)=(F, B)$ is soft $\beta$-open and non empty. We have that $(\mathrm{F}, \mathrm{A})$ is a non empty proper soft $\beta$-clopen set in (F,E). Which shows that (2) is false. Therefore $(2) \Rightarrow(3)$ 
(3) $\Rightarrow(4)$ It is obvious.

(4) $\Rightarrow(5)$

If (5) is false, then $(F, E)=(F, A) \cup(F, B)$, where (F, A) and ( F, B) are non empty and soft $\beta$-separated sets, since $(F, A) \cap \underline{(F, B)_{\beta}}=\phi$. We conclude that $(F, B)_{\beta} \subseteq(F, B)$, so $(\mathrm{F}, \mathrm{B})$ is soft $\beta$-closed. Similarly, (F, A) must be soft $\beta$-closed. Therefore (4) fails. Therefore (4) $\Rightarrow(5)$.

$(5) \Rightarrow(1)$

Suppose (1) is false and that (F, A) is a non empty proper soft $\beta$-clopen subset of $(\mathrm{F}, \mathrm{E})$. Then $(F, B)=(F, E)-(F, A)$ is non empty and soft $\beta$-clopen. So $(\mathrm{F}, \mathrm{A})$ and $(\mathrm{F}, \mathrm{B})$ are soft $\beta$-separated sets. Since $(F, E)=(F, A) \cup(F, B)$. Which shows that (5) is false. Therefore $(5) \Rightarrow(1)$.

Theorem 4.2. Let $(\mathrm{X}, \tau, \mathrm{E})$ be a soft topological space and let $(\mathrm{F}, \mathrm{A})$ be a soft $\beta$-connected. Let (F, B) and (F, C) are soft $\beta$-separated sets. If $(F, A) \subseteq(F, B) \cup(F, C)$. Then either $(F, A) \subseteq(F, B)$ or $(F, A) \subseteq(F, C)$.

Proof: Suppose (F, A) is soft $\beta$-connected and $(\mathrm{F}, \mathrm{B}),(\mathrm{F}, \mathrm{C})$ are soft $\beta$-separated sets such that $(F, A) \subseteq(F, B) \cup(F, C)$. Let $(F, A) \not \subset(F, B)$ and $(F, A) \not \subset(F, C)$.

Suppose $\quad\left(F, A_{1}\right)=(F, B) \cap(F, A) \neq \phi \quad$ and $\quad\left(F, A_{2}\right)=(F, C) \cap(F, A) \neq \phi . \quad$ Then $(F, A)=\left(F, A_{1}\right) \cup\left(F, A_{2}\right)$. Since $\left(F, A_{1}\right) \subseteq(F, B)$. Hence $s \beta c l\left(F, A_{1}\right) \subseteq s \beta c l(F, B)$. Since $s \beta c l(F, B) \cap(F, C)=\phi \quad$ then $\quad s \beta c l\left(F, A_{1}\right) \cap\left(F, A_{2}\right)=\phi$. Since $\quad\left(F, A_{2}\right) \subseteq(F, C)$. Hence $s \beta c l\left(F, A_{2}\right) \subseteq s \beta c l(F, C)$. Since $s \beta c l(F, C) \cap(F, B)=\phi$. Then $s \beta c l\left(F, A_{2}\right) \cap\left(F, A_{1}\right)=\phi$. But $(F, A)=\left(F, A_{1}\right) \cup\left(F, A_{2}\right)$, therefore $(\mathrm{F}, \mathrm{A})$ is not soft $\beta$-connected space. This is contradiction. Then either $(F, A) \subseteq(F, B)$ or $(F, A) \subseteq(F, C)$.

Theorem 4.3. If $(\mathrm{F}, \mathrm{A})$ is soft $\beta$-connected set then $\operatorname{sicl}(F, A)$ is soft $\beta$-connected.

Proof: Suppose (F, A) is soft $\beta$-connected set and $\operatorname{sicl}(F, A)$ is not. Then there exists two soft $\beta$-separated sets $(\mathrm{F}, \mathrm{B})$ and $(\mathrm{F}, \mathrm{C})$ such that $s \beta c l(F, A)=(F, B) \cup(F, C)$. But $(F, A) \subseteq s \beta c l(F, A)$, then $(F, A)=(F, B) \cup(F, C)$ and since $(\mathrm{F}, \mathrm{A})$ is soft $\beta$-connected set then by above theorem 4.2 either $(F, A) \subseteq(F, B)$ or $(F, A) \subseteq(F, C)$.

(i). If $(F, A) \subseteq(F, B)$ then $s \beta c l(F, A) \subseteq s \beta c l(F, B)$. But $s \beta c l(F, B) \cap(F, C)=\phi$. Hence $s \beta c l(F, A) \cap(F, C)=\phi$. Since $(F, C) \subseteq s \beta c l(F, A)$, then $(F, C)=\phi$. This is contradiction.

(ii). If $(F, A) \subseteq(F, C)$ then the same way we can prove that $(F, B)=\phi$ which is contradiction. Therefore $\operatorname{sicl}(F, A)$ is soft $\beta$-connected.

Theorem 4.4. If $(\mathrm{F}, \mathrm{A})$ is soft $\beta$-connected set and $(F, A) \subseteq(F, B) \subseteq s \beta c l(F, A)$ then $(\mathrm{F}, \mathrm{B})$ is soft $\beta$-connected.

Proof: If (F, B) is not soft $\beta$-connected, then there exists two soft sets (F, C) and (F, D) such that $s \beta c l(F, C) \cap(F, D)=(F, C) \cap s \beta c l(F, D)=\phi$ and $(F, B)=(F, C) \cup(F, D)$.

Since $(F, A) \subseteq(F, B)$, thus either $\quad(F, A) \subseteq(F, C) \quad$ or $\quad(F, A) \subseteq(F, D)$. Suppose $(F, A) \subseteq(F, C)$ then $s \beta c l(F, A) \subseteq s \beta c l(F, C)$, thus $s \beta c l(F, A) \subseteq(F, D)=s \beta c l(F, C) \cap(F, D)=\phi$.

But $(F, D) \subseteq(F, B) \subseteq s \beta c l(F, A)$ thus $s \beta c l(F, A) \cap(F, D)=(F, D)$. Therefore $(F, D)=\phi$ which is contradiction. Thus (F, B) is soft $\beta$-connected. If $(F, A) \subseteq(F, B)$, then similarly we can prove that $(F, C)=\phi$. This is contradiction. Then $(\mathrm{F}, \mathrm{B})$ is soft $\beta$-connected. 
Definition 4.5. Let $(\mathrm{X}, \tau, \mathrm{E})$ be a soft topological space over $\widetilde{X}$. Then soft $\beta$-boundary of soft set $(\mathrm{H}, \mathrm{E})$ over $\tilde{X}$ is denoted by $(H, E)_{\beta}$ and is defined as $(H, E)_{\beta}=\overline{(H, E)} \cap \overline{\left((H, E)^{\prime}\right)}$, obviously $(H, E)_{\beta}$ is smallest soft closed set over $\tilde{X}$ containing $(\mathrm{H}, \mathrm{E})$.

Theorem 4.6. Let $(\mathrm{X}, \tau, \mathrm{E})$ be soft topological space and $(\mathrm{F}, \mathrm{E})$ be soft subset of $\tilde{X}$. Then, $(F, E) \cup \underline{(F, E)^{\beta}}=\overline{(F, E)}$.

Proof: If $e_{x} \in(F, E) \cup \underline{(F, E)_{\beta}}$, then $e_{x} \in(F, E)$ or $e_{x} \in \underline{(F, E)_{\beta}}$. If $e_{x} \in(F, E)$, then $e_{x} \in \operatorname{sicl}(F, E)$ if $e_{x} \in(F, E)_{\beta}$ then $(G, E) \cap\left((F, E)-e_{x}\right) \neq \phi$, for all soft $\beta$-open neighborhoods $(\mathrm{G}, \mathrm{E})$ of $e_{x}$, and so $(G, E) \cap(F, E) \neq \phi$, for all soft $\beta$-open neighborhoods (G, E) of $e_{x}$, and so $(G, E) \cap(F, E) \neq \phi$, for all soft $\beta$-open neighborhoods $(\mathrm{G}, \mathrm{E})$ of $e_{x}$; hence, $e_{x} \in \operatorname{s} \beta c l(F, E)$.

Conversely, if $e_{x} \in \operatorname{sicl}(F, E)$, then $e_{x} \in(F, E)$ or $e_{x} \notin(F, E)$. If $e_{x} \in(F, E)$, it is trivial that $e_{x} \in(F, E) \cup(F, E)_{\beta}$, if $e_{x} \notin(F, E)$, then $(G, E) \cap\left((F, E)-e_{x}\right) \neq \phi$, for all soft $\beta$-open neighborhoods $(\mathrm{G}, \mathrm{E})$ of $e_{x}$. Therefore, $e_{X} \in \underline{(F, E)_{\beta}}$ implies $e_{x} \in(F, E) \cup \underline{(F, E)_{\beta}}$. So, $(F, E) \cup(F, E)_{\beta}=s \beta c l(F, E)$. Hence the proof.

Theorem 4.7. Let $(\mathrm{X}, \tau, \mathrm{E})$ be a soft topological space and $(\mathrm{H}, \mathrm{E}),(\mathrm{K}, \mathrm{E})$ are soft $\beta$-open subsets of X. Then,
(1) $(H, E) \subseteq(K, E) \Rightarrow(H, E)^{\beta d} \subseteq(K, E)^{\beta d}$
(2) $((H, E) \cap(K, E))^{\beta d} \subseteq(H, E)^{\beta d} \cap(K, E)^{\beta d}$
(3) $((H, E) \cup(K, E))^{\beta d} \subseteq(H, E)^{\beta d} \cup(K, E)^{\beta d}$
(4) $\left((H, E)^{\beta d}\right)^{\beta d} \subseteq(H, E)^{\beta d}$
(5) $\overline{(H, E)^{\beta d}}=(H, E)^{\beta d}$

Proof: Let $(H, E) \subseteq(G, E)$. Since $(H, E)-x \subseteq(K, E)-x, \Rightarrow \overline{(H, E)-x}^{\beta} \subseteq \overline{(K, E)-x}^{\beta}$, then $(H, E)^{\beta d} \subseteq(K, E)^{\beta d}$.

(2) $(H, E) \cap(K, E) \subseteq(H, E)$ and $(H, E) \cap(K, E) \subseteq(K, E)$. Then by result (1), we have $((H, E) \cap(K, E))^{\beta d} \subseteq(H, E)^{\beta d} \quad$ and $\quad((H, E) \cap(K, E))^{\beta d} \subseteq(K, E)^{\beta d}$. Therefore $((H, E) \cap(K, E))^{\beta d} \subseteq(H, E)^{\beta d} \cap(K, E)^{\beta d}$.

(3) For all $e_{x} \in((H, E) \cup(K, E))^{\beta d}$ implies $e_{x} \in \overline{((H, E) \cup(K, E))-\left\{e_{x}\right\}}$. Therefore

$$
\begin{aligned}
& \overline{((H, E) \cup(K, E))-\left\{e_{x}\right\}^{\beta}}=\overline{((H, E) \cup(K, E)) \cap\left\{e_{x}\right\}^{c}}{ }^{\beta} \\
& =\overline{\left((H, E) \cap\left\{e_{x}\right\}^{c}\right) \cup(K, E) \cap\left\{e_{x}\right\}^{c}} \beta \\
& =\overline{\left((H, E) \cap\left\{e_{x}\right\}^{c}\right)} \cup \overline{\left((H, E) \cap\left\{e_{x}\right\}^{c}\right)} \\
& =\overline{\left((H, E)-\left\{e_{x}\right\}\right)} \cup \overline{\left((H, E)-\left\{e_{x}\right\}\right)} \text {. }
\end{aligned}
$$

By Theorem 1 of [17] if and only if $e_{x} \in(H, E)^{\beta d} \cup(K, E)^{\beta d}$. Hence $((H, E) \cup(K, E))^{\beta d}=(H, E)^{\beta d} \cup(K, E)^{\beta d}$.

(4) Suppose $e_{x} \notin(H, E)^{\beta d}$. Then $e_{x} \notin{\overline{(H, E)-e_{x}}}^{\beta}$. This implies that there is a soft $\beta$-open set $(\mathrm{K}, \mathrm{E})$ such that $(H, E) \cap\left((K, E)-\left\{e_{x}\right\}\right)=\phi$. Then $e_{x} \in \overline{(F, E)^{\beta d}}-\left\{e_{x}\right\}$. Since $e_{x} \in(K, E)$, we have 
$(K, E) \cap \overline{(H, E)^{\beta d}-\left\{e_{x}\right\}} \neq \phi$. Therefore, there is $e_{y} \neq e_{x}$ such that $e_{y} \in(K, E) \cap(H, E)^{\beta d}$. It follows that $e_{y} \in\left((K, E)-\left\{e_{x}\right\}\right) \cap\left((H, E)-\left\{e_{y}\right\}\right)$. Hence $\quad\left((K, E)-\left\{e_{x}\right\}\right) \cap\left((H, E)-\left\{e_{y}\right\}\right) \neq \phi, \quad$ a contradiction to the fact that $(K, E) \cap\left((H, E)-\left\{e_{x}\right\}\right)=\phi$. This implies that $e_{x} \in\left((H, E)^{\beta d}\right)^{\beta d}$ and so $\left((H, E)^{\beta d}\right)^{\beta d} \subseteq(H, E)^{\beta d}$.

(5) By the results (2),(3),(5) and by the above Theorem 4.6, we have completed the proof.

Theorem 4.8. If the soft sets $(\mathrm{L}, \mathrm{A})$ and $(\mathrm{G}, \mathrm{C})$ form a soft $\beta$-separation of $(\mathrm{U}, \mathrm{E})$, and if $(\mathrm{V}, \mathrm{B})$ is a soft $\beta$-connected subspace of $(\mathrm{U}, \mathrm{E})$, then $(V, B) \subseteq(L, A)$ or $(V, B) \subseteq(G, C)$.

Proof: Since (L, A) and $(\mathrm{G}, \mathrm{C})$ are disjoint soft $\beta$-open sets, so are $(L, A) \cap(V, B)$, and $(G, C) \cap(V, B)$ and their soft gives $(\mathrm{V}, \mathrm{B})$ that is there will be soft $\beta$-separation of $(\mathrm{V}, \mathrm{B})$, a contradiction. Hence, one of $(L, A) \cap(V, B)$ and $(G, C) \cap(V, B)$ is empty and so $(\mathrm{V}, \mathrm{B})$ is entirely contained in one of them.

Theorem 4.9. The soft union $(\mathrm{F}, \mathrm{A})$ of any family $\left\{\left(F, A_{i}\right): i \in I\right\}$ of soft $\beta$-connected having a non empty soft intersection is a soft $\beta$-connected.

Proof: Let (F, A) be a soft union of any family of soft $\beta$-connected sets having a non empty soft intersection. Suppose $(F, A)=(F, B) \cup(F, C)$, where $(\mathrm{F}, \mathrm{B})$ and $(\mathrm{F}, \mathrm{C})$ form a soft $\beta$-separation of (F, A) by hypothesis, we may choose a soft point $\left(x_{f_{A_{i}}}\right)=\left(x, f_{A_{i}}(x)\right) \in \cap_{i \in I}\left(F, A_{i}\right)$. Then $\left(x_{f_{A_{i}}}\right)$ must belong to either a soft subset of (F, B) or a soft subset of (F, C). Since (F, B) and (F, C) are soft disjointed, we must have $\left(F, A_{i}\right) \subseteq(F, B)$ for all $i \in I$, and so $(F, A) \subseteq(F, B)$. From this we obtain that $(F, C)=(F, \phi)$. Which is contradiction. This proves the theorem.

Theorem 4.10. If a soft topological space $(\mathrm{X}, \tau, \mathrm{E})$ contains a soft $\beta$-connected subspace $\left(F, E_{1}\right)$ such that $\operatorname{sicl}\left(F, E_{1}\right)=(F, E)$, then $(\mathrm{X}, \tau, \mathrm{E})$ is soft $\beta$-connected.

Proof: Suppose $\left(F, E_{1}\right)$ is soft $\beta$-connected subspace of $(\mathrm{X}, \tau, \mathrm{E})$ such that $\operatorname{sicl}\left(F, E_{1}\right)=(F, E)$. Since $\left(F, E_{1}\right) \subseteq(F, E)=s \beta c l\left(F, E_{1}\right)$ then by theorem 4.8, $(\mathrm{X}, \tau, \mathrm{E})$ is soft $\beta$-connected.

Theorem 4.11. If $\left(X, \tau_{1}, E_{1}\right)$ and $\left(Y, \tau_{2}, E_{2}\right)$ are soft $\beta$-connected subspace of soft topological space $(\mathrm{X}, \tau, \mathrm{E})$ such that $\left(F_{E_{1}}\right) \cap\left(F_{E_{2}}\right) \neq(F, \phi)$ then $\left(F, E_{1}\right) \cup\left(F, E_{2}\right)$ is a soft $\beta$-connected subspace.

Proof: Suppose $\left(F, E_{1}\right) \cup\left(F, E_{2}\right)$ is not soft $\beta$-connected subspace. Then there exists two soft $\beta$-separated sets $(\mathrm{F}, \mathrm{A})$ and $(\mathrm{F}, \mathrm{B})$ such that $\left(F, E_{1}\right) \cup\left(F, E_{2}\right)=(F, A) \cup(F, B)$. Since $\left(F, E_{1}\right) \subseteq\left(F, E_{1}\right) \cup\left(F, E_{2}\right)=(F, A) \cup(F, B)$ and $\left(F, E_{1}\right)$ is soft $\beta$-connected then by theorem 4.3 either $\left(F, E_{1}\right) \subseteq(F, A)$ or $\left(F, E_{1}\right) \subseteq(F, B)$. Since $\left(F, E_{2}\right) \subseteq\left(F, E_{1}\right) \cup\left(F, E_{2}\right)=(F, A) \cup(F, B)$ and $\left(F, E_{2}\right)$ is soft $\beta$-connected, then either $\left(F, E_{2}\right) \subseteq(F, A)$ or $\left(F, E_{2}\right) \subseteq(F, B)$.

(i) if $\left(F, E_{1}\right) \subseteq(F, A)$ and $\left(F, E_{2}\right) \subseteq(F, B)$, then $\left(F, E_{1}\right) \cup\left(F, E_{2}\right) \subseteq(F, A)$. Hence $(F, B)=(F, \phi)$. This is contradiction.

(ii) if $\left(F, E_{1}\right) \subseteq(F, A)$ and if $\left(F, E_{2}\right) \subseteq(F, B)$, then $\left(F, E_{1}\right) \cap\left(F, E_{2}\right) \subseteq(F, A) \cap(F, B)=(F, \phi)$. Therefore $\left(F, E_{1}\right) \cap\left(F, E_{2}\right)=(F, \phi)$ which is contradiction. Then by following the same procedure we get a contradiction if $\left(F, E_{1}\right) \subseteq(F, B)$ and $\left(F, E_{2}\right) \subseteq(F, A)$ or if $\left(F, E_{1}\right) \subseteq(F, A)$ and $\left(F, E_{2}\right) \subseteq(F, B)$. Therefore $\left(F, E_{1}\right) \cup\left(F, E_{2}\right)$ is soft $\beta$-connected subspace of $(\mathrm{X}, \tau, \mathrm{E})$. 
Theorem 4.12. Let $\left(X, \tau_{1}, E\right)$ be a soft $\beta$-connected space and $\tau_{2} \subseteq \tau_{1}$. Then $\left(X, \tau_{2}, E\right)$ is soft $\beta$-connected.

Proof: Suppose on the contrary (F, A) and $(\mathrm{F}, \mathrm{B})$ is a soft $\beta$-separation of $(\mathrm{F}, \mathrm{E})$ with soft topology $\tau_{2}$. Since $\tau_{2} \subseteq \tau_{1}$ then (F, A) and (F, B) is a soft $\beta$-separation of (F, B) with soft topology $\tau_{1}$. This is contradiction. Therefore $\left(X, \tau_{2}, E\right)$ is soft $\beta$-connected.

Theorem 4.13. Let $(X, \tau, E)$ and $\left(Y, \tau^{\prime}, E^{\prime}\right)$ be two soft topological spaces and $u: X \rightarrow Y$ and $p: E \rightarrow E^{\prime}$ be mappings. Also a soft mapping $f_{p u}: S S(X)_{E} \rightarrow S S(Y)_{E^{\prime}}$ is soft pu-continuous and soft onto. If $(\mathrm{X}, \tau, \mathrm{E})$ is soft $\beta$-connected, then the soft image of $(\mathrm{X}, \tau, \mathrm{E})$ is also soft $\beta$-connected.

Proof: Let a soft mapping $f_{p u}: S S(X)_{E} \rightarrow S S(Y)_{E}$, be soft pu continuous and soft onto. On the contrary, suppose that $\left(Y, \tau^{\prime}, E^{\prime}\right)$ is soft $\beta$-connected and pair $\left(H_{1}, E^{\prime}\right)$ and $\left(H_{2}, E^{\prime}\right)$ is soft $\beta$-disconnection of $\left(Y, \tau^{\prime}, E^{\prime}\right)$. Since $f_{p u}: S S(X)_{E} \rightarrow S S(Y)_{E^{\prime}}$ is soft pu-continuous, therefore $f_{p u}^{-1}\left(H_{1}, E^{\prime}\right)$ and $f_{p u}^{-1}\left(H_{2}, E^{\prime}\right)$ is a soft $\beta$-disconnection of $(X, \tau, E)$, which is contradiction. Hence $\left(Y, \tau^{\prime}, E^{\prime}\right)$ is soft $\beta$-connected. This completes the proof.

\section{Conclusion}

The study of soft sets and soft topology is very important during the study towards applications in non-classical and classical logic. Here, we defined and explored the properties of soft $\beta$-connected spaces in soft topological spaces and discussed the behaviour of soft $\beta$-connected spaces in soft topological spaces under soft pu-continuous mappings. We have also characterized soft $\beta$-connectedness in terms of soft $\beta$-boundary and discussed some more properties. We expect that the findings in this paper can be applied to problems of many fields that contains uncertainties and will enhance the further study on soft topology to carry out general framework for the applications in practical life.

\section{Acknowledgements}

The authors are grateful to the University Grants Commission, New Delhi, India for its financial support by UGC-SAP DRS-III under F-510/3/DRS-III/2016(SAP-I) dated 29th Feb 2016 to the Department of Mathematics, Karnatak University, Dharwad-580003, India. Also this research was supported by the University Grants Commission, New Delhi, India, under no F1-17.1/201314/MANF-2013-14-MUS-KAR-22545.

\section{References}

[1] L.A. Zadeh, Fuzzy sets, Inf. Control. 8(3) (1965) 338-353.

[2] K. Atanassov, Operators over interval valued intuitionistic fuzzy sets, Fuzzy Sets and Systems. 64 (1994) 159-174.

[3] K. Atanassov, Intuitionistic fuzzy sets, Fuzzy Sets and Systems. 20(1) (1986) 87-96.

[4] Z. Pawlak, Rough sets, Int. J. Comput. Sci. 11 (1987) 341-356.

[5] C.H. Park, Y.B. Jun, M.A. Ozturk, Soft WS-algebras, Commun. Korean Math. Soc. 23(3) (2008) 313-324.

[6] D. Molodtsov, Soft set theory - first results, Comput. Math. Appl. 37(4-5) (1999) 19-31. 
[7] D. Molodtsov, V.Y. Leonov, D.V. Kovkov, Soft sets technique and its application, Nechetkie Sistemy i Myagkie Vychisleniya. 1(1) (2006) 8-39.

[8] P.K. Maji, R. Biswas, R. Roy, An application of soft sets in a decision making problem, Comput. Math. Appl. 44(8-9) (2002) 1077-1083.

[9] P.K. Maji, R. Biswas, R. Roy, Soft set theory, Comput. Math. Appl. 45(4-5) (2003) 555-562.

[10] M. Shabir, M. Naz, On soft topological spaces, Computers and Math. with Appl. 61(7) (2011) 1786-1799.

[11] S. Hussain, B. Ahmad, Some properties of soft topological spaces, Comput. Math. Appl. 62(11) (2011) 4058-4067.

[12] B. Ahmad, S. Hussain, On some structures of soft topology, Math. Sci. 64(6) (2012).

[13] A. Kharral, B. Ahmad, Mappings on soft classes, New Math. Nat. Comput. 7(3) (2011) 471481.

[14] I. Zorlutuna et al., Remarks on soft topological spaces, Annals of Fuzzy Mathematics and Informatics. 3(2) (2011) 171-185.

[15] M. Akdag, A. Ozkan, Soft b-open sets and soft b-continuous functions, Math. Sci. 8(2) (2014) 124.

[16] V. Seenivasan, S. Kalaiselvi, Soft gsg-closed set in soft topological spaces, International Journal of Mathematics Trends and Technology. 4(10) (2013) 196-200.

[17] I. Arockiarani, A. Arokialancy, Generalized soft $\mathrm{g} \beta$-closed sets and soft gs $\beta$-closed sets in soft topological spaces, International Journal of Mathematical Archive. 4(2) (2013) 1-7.

[18] J. Subhashinin, C. Sekar, Soft P-connectedness via soft P-open sets, International Journal of Mathematics Trends and Technology. 6(3) (2014) 203-214.

[19] B. Chen, Some local properties of soft semi-open sets, Discrete Dynamics in Nature and Society. 2013 (2013).

[20] S.S. Benchalli, P.G. Patil, A.S. Dodamani, Some properties of soft $\beta$-separation axioms in soft topological spaces, International Journal of Scientific and Innovative Mathematical Research. 3(1) (2015) 254-259.

[21] S.S. Benchalli, P.G. Patil, A.S. Dodamani, Soft $\beta$-compactness in soft topological spaces, Mathematical Sciences. International Research Journal. 4(2) (2015) 214-218.

[22] S.S. Benchalli, P.G. Patil, A.S. Dodamani, Soft $\beta$-locally closed spaces in soft topological spaces, Asian Journal of Mathematics and Computer Research. 14(3) (2016).

[23] S.S. Benchalli, P.G. Patil, N. Kabbur, On soft $\gamma$-operations in soft topological spaces, Journal of New Theory. 28(3) (2015) 223-235.

[24] S.S. Benchalli, P.G. Patil, N. Kabbur, On some weaker forms of soft closed sets in soft topological spaces, International Journal of Applied Mathematics. 6 (2015) 20-32.

[25] M. Akdag, A. Ozkan, Soft $\alpha$-open sets and soft $\alpha$-continuous functions, Abstract and Applied Analysis. 2014 (2014).

[26] M. Akdag, A. Ozkan, On soft $\beta$-open sets and soft $\beta$-continuous functions, The Scientific World Journal. 4 (2014) 1-6. 